\title{
Expansión de la ciudad turistica y nuevas resistencias. El caso de Airbnb en Madrid
}

\section{The expansion of the tourist city and new resistances. The case of Airbnb in Madrid}

\author{
JAVIER GIL \\ Universidad Nacional de Educación a Distancia \\ jagil@poli.uned.es (ESPAÑA) \\ Jorge SEQUERA \\ Universidade Nova de Lisboa (PORTUGAL)
}

Recibido: 05.042018
Aceptado: 31.07 .2018

\section{RESUMEN}

La reciente y veloz expansión de la turistificación ofrece algunos factores críticos que han alimentado la indignación vecinal y la resistencia local dentro de la 'ciudad turistística'. Estas protestas están muy centradas en los impactos negativos de la turistificación en el mercado inmobiliario local, en la construcción de la ciudad para el monocultivo del turismo, en la expulsión de familias de barrios turistificados y en el deterioro de la convivencia vecinal. De este modo, diferentes actores locales y grupos sociales muestran un abanico de historias no lineales y discursos diversos sobre los impactos de la reciente y veloz expansión del turismo urbano (in)formal en sus comunidades locales. En este artículo, en primer lugar, se analiza el desarrollo de la actividad de Airbnb en la ciudad de Madrid. Los resultados reflejan que el mercado de Airbnb en la ciudad de Madrid está controlado por actores profesionales que no cumplen los principios de la economía colaborativa. Su actividad se basa en aprovechar los "rent gaps" que genera Airbnb y ampliar las rentas inmobiliarias por medio de sustituir vivienda residencial en vivienda para turísticas, lo que impulsa los procesos de turistificación. Este proceso está generando malestar entre los ciudadanos y está dando lugar a nuevas resistencias contra la turistización de la ciudad. En segundo lugar, el artículo analiza las respuestas sociales que se está dando a la reciente turistificación urbana que ha tenido lugar en el centro de la ciudad de Madrid, 
prestando especial atención a la variedad de actores políticos, de estrategias y de acción colectiva en el centro de Madrid a lo largo de los últimos años. Este artículo busca por tanto analizar cómo se desarrolla la turistificación a través de Airbnb, para después ofrecer un análisis del enfoque con que los movimientos sociales urbanos luchan por una coexistencia urbana inclusiva en la compleja 'ciudad turística'.

\title{
PALABRAS CLAVE
}

Turistificación, Airbnb, derecho a la ciudad, ciudad turística.

\begin{abstract}
The recent and rapid expansion of touristification offers some critical factors that have fueled the local indignation and local resistance within the 'tourist city'. These protests are very focused on the negative impacts of touristification on the local real estate market, on the construction of the city for the monoculture of tourism, on the evictions of families from touristized neighborhoods and on the deterioration of neighborhood coexistence. In this way, different local actors and social groups show a range of non-linear histories and diverse discourses about the impacts of the recent and rapid expansion of urban (in) formal tourism in their local communities. In this article, in the first place, we analyze the expansion of Airbnb in the city of Madrid. The results reflect that the Airb$\mathrm{nb}$ market in the city of Madrid is controlled by professional actors who do not comply with the principles of the sharing economy. Its activity is based on taking advantage of the «rent gaps» generated by Airbnb and expanding real estate income by transforming residential housing into short-term rentals for tourists, which drives up touristification processes. This process is generating discomfort among citizens and is giving rise to new resistances against the touristization of the city. In second place, the article analyzes the social responses that are taking place against the recent urban touristification that has taken place in the center of the city of Madrid, paying special attention to the variety of political actors, strategies and collective actions in the center of Madrid over the past years. This article seeks to analyze how touristification is expanding through Airbnb, and to offer an analysis of the approach with which urban social movements fight for an inclusive urban coexistence in the complex 'tourist city'.
\end{abstract}

\section{KEY WORDS}

Touristification, Airbnb, right to the city, the 'tourist city'. 


\section{INTRODUCCIÓN}

La reciente turistificación de los barrios del centro histórico de muchas ciudades europeas, principalmente basada en la expansión del sector (in)formal de alojamiento turístico (Gottlieb, 2013), está provocando un fuerte impacto social, espacial y económico. Especialmente en Europa, la revalorización socio-espacial de los barrios del centro histórico de muchas ciudades desde mediados de la década de 1980 ha contribuido con fuerza a transformar la ciudad en una zona de consumo orientada al ocio (Wynne \& O'Connor, 1998; Ritzer, 2010). Así, el turismo urbano en los últimos años ha jugado un papel clave en este proceso de 'ludificación de la ciudad' (Baptista, 2005). Desde una perspectiva más amplia, el proceso de turistificación conlleva una serie de impactos, como: (a) el incremento de tensiones en los mercados inmobiliarios locales, causando un creciente desplazamiento espacial de ciertas capas de la población (Pixová \& Sládek, 2016); (b) la desposesión simbólica y material de los vecinos provocada por la rápida turistificación de sus barrios (Janoschka \& Sequera, 2016); c) la creciente marginalización de presencias, prácticas y consumos 'no deseados' mediante políticas gubernamentales de tolerancia-cero (Cummings, 2015; Sequera, 2017); (d) la promoción de nuevas formas de ocio hedonista, mercantilizado y juvenil durante las horas nocturnas, lo que genera tensiones en la convivencia dentro de la comunidad (Nofre et al, 2018); (e) la desaparición de la tradicional venta al por menor y la manufactura, reemplazadas por negocios orientados al turismo (González \& Waley, 2013); y finalmente, (f) la relación entre 'turistificación urbana' (Ashworth \& Page, 2011), las crisis de vivienda (Lee, 2016; Merante y Horn, 2016) y otros procesos urbanos como la gentrificación o la implementación de 'políticas de gentrificación' (Sequera, 2013, 2015, 2017; Wachsmuth y Weisler, 2017).

Asimismo, la veloz expansión de la turistificación ha alimentado la indignación vecinal y la resistencia local dentro de la 'ciudad turistística' (Colomb $\&$ Novy, 2017). En este sentido, diversos autores sugieren que la reciente turistificación de las zonas del centro de la ciudad viene acompañada a menudo de procesos de desplazamiento de habitantes del barrio, lo que está dando origen a un aumento de las protestas vecinales (p. ej., Gotham, 2005; Pareja-Eastaway \& Simó, 2014; Cócola-Gant, 2016). Estas protestas están muy centradas en los impactos negativos de la turistificación en el mercado inmobiliario local, en el modelo de ciudad basada en el monocultivo del turismo (Conti \& Perelli, 2007), o en la preocupación por la expulsión de familias de barrios turistificados o el deterioro de la convivencia vecinal (Nofre et al, 2018).

Si bien el peso de la economía del turismo estaba haciendo pensar hasta hace muy poco tiempo que el turismo contribuía significativamente tanto a revitalizar los barrios históricos degradados como a proporcionar empleo, el debate en el último año se ha polarizado. Por un lado, el estatus del turismo y el ocio en muchas ciudades europeas ha cambiado radicalmente a ojos de los legisladores urbanos y las élites empresariales hoteleras. Por otro, este relato del mercado está siendo socialmente contestado. Diferentes actores locales y grupos sociales 
están mostrando los impactos negativos de la expansión del turismo urbano (in) formal en sus comunidades locales.

En este artículo trataremos de analizar las respuestas sociales que se está dando a la reciente turistificación que ha tenido lugar en el centro de la ciudad de Madrid, prestando especial atención a la variedad de actores políticos y de estrategias de acción colectiva en el centro de Madrid a lo largo de los últimos años. Este artículo por tanto quiere ofrecer un análisis del enfoque con que los movimientos sociales urbanos luchan por una coexistencia urbana inclusiva en la compleja "ciudad turística'.

\section{AIRBNB Y LA PROFESIONALIZACIÓN DE LA ECONOMÍA COLABORATIVA}

El rápido crecimiento y cada vez mayor penetración de la plataforma Airbnb en la mayoría de ciudades del mundo, está generando fuertes conflictos y un malestar generalizado, ya que el desarrollo del mercado de pisos turísticos está aumentando los impactos negativos de la turistificación. Sobre la plataforma se articulan toda una serie de actividades heterogéneas que adoptan diversa formas, que tienen distintos efectos sobre la ciudad, la economía y la turistificación en general. En investigaciones previas (Gil, 2018) se ha señalado que en Airbnb existen distintos tipos de anfitriones: i. Anfitriones esporádicos: personas que hospedan de manera puntual a lo largo del año en su primera residencia; ii. Anfitriones permanentes: personas que hospedan de manera continua a lo largo del año, en su primera residencia; iii. Anfitriones profesionales: personas que alquilan viviendas en el mercado residencial, para subalquilar las habitaciones o la vivienda en Airbnb, y; iv. Anfitriones inversores: personas que extraen vivienda del mercado residencial para alquilarla en Airbnb.

Todo proyecto de "economía colaborativa" tiene que cumplir un principio fundamental: que el recurso que se moviliza sea un bien temporalmente infrautilizado (Botsman y Rogers, 2011; Gordo, Rivera y Cassidy, 2017; Rodríguez, 2017). Es decir, ser un bien temporalmente ocioso. En el caso de Airbnb, se trataría de alquilar la vivienda personal mientras no está siendo utilizada (por ejemplo, cuando el inquilino se va de vacaciones). Pero en la práctica se observa que en Airbnb no todas las ofertas de hospedaje cumplen este principio (Gil, 2018). Los anfitriones esporádicos y permanentes sí que cumplen este principio y su actividad sí que responde al modelo de economía colaborativa (modelo $\mathrm{p} 2 \mathrm{p}$ ), ya que se trata de particulares que realizan la actividad en su primera residencia para obtener un ingreso extra. Por el contrario, los anfitriones profesionales e inversores no cumplen con este principio básico de las economías colaborativas, ya que su actividad consiste en extraer vivienda del mercado residencial para introducirla en Airbnb y alquilarla de manera continua a lo largo del año. Esta actividad no genera ingresos extras sino que se trata de un negocio propio

${ }^{1}$ El trabajo que figura a continuación se llevó a cabo durante 2016 y 2017 
de la economía tradicional. Además se observa que detrás de esta actividad se encuentran inmobiliarias, empresas especializadas, así como grandes y pequeños propietarios de vivienda. Por lo tanto, este tipo de actividad no responde al modelo $\mathrm{p} 2 \mathrm{p}$, sino a un modelo de hospedaje comercial que se desarrolla sobre una plataforma de hospedaje entre particulares ( $22 p)$.

Diferenciar entre la actividad en Airbnb que responde al modelo $\mathrm{p} 2 \mathrm{p}$ y la que responde al modelo comercial, es fundamental porque el impacto de ambos tipos sobre los procesos de turistización varían de manera considerable de un caso a otro. Cuanto mayor sea el peso del modelo comercial en una ciudad, mayor será su impacto sobre los procesos de turistificación. En cambio, cuanto mayor sea el peso del modelo $\mathrm{p} 2 \mathrm{p}$ en una ciudad, menor será su impacto sobre los procesos de turistificación.

En estudios previos que analizan el impacto de Airbnb sobre la ciudad según la actividad responda al modelo p2p o al modelo comercial (Gil, 2018), se observa que a nivel internacional el mercado de Airbnb está controlado principalmente por anfitriones que responden al modelo comercial. Se trata de anfitriones multipropietarios que gestionan múltiples ofertas en la plataforma, que en ningún caso son primeras residencias, y que en la mayoría de casos su actividad consiste en extraer viviendas del mercado residencial para introducirlas en Airbnb, siendo las ofertas del modelo p2p una minoría (Gil, 2018 ). Así, para el caso de Madrid, nos encontramos que es una de las ciudades del mundo donde el porcentaje de ofertas $\mathrm{p} 2 \mathrm{p}$ es menor y el número de multipropietarios es mayor, en contraposición a ciudades como Copenhague, Berlín, Ámsterdam, Nueva York, Sídney o San Francisco. Madrid se caracteriza por tener un mercado de Airbnb en donde muy pocos actores (empresas especializadas, inmobiliarias y grandes propietarios) controlan la mayoría del mercado.

De este modo, podemos observar que en Madrid, un 75,79\% de los anfitriones en Airbnb responden al modelo $\mathrm{p} 2 \mathrm{p}$ (anfitriones esporádicos y permanentes), que tienen un único anuncio en la plataforma, y los anfitriones que responden al modelo comercial (profesionales e inversores), con múltiples anuncios en la plataforma, tan solo representan al 24,21\% de los anfitriones. Estos datos parecen señalar que en Madrid la actividad de Airbnb responde a un modelo de hospedaje en que los particulares hospedan en su primera residencia bajo los principios de la economía colaborativa (modelo p2p), como anuncia la propia empresa (Airbnb, 2018). Pero al analizar los datos en profundidad, se observa una contradicción: en Madrid, la mayoría de anfitriones responden al modelo p2p, pero los actores comerciales, que solo representan al 24,21\% de los anfitriones, controlan la mayoría de pisos el alquiler. Es decir, un pequeño grupo de usuarios comerciales controlan la mayoría de las ofertas de hospedaje en Airbnb.

El mercado de anfitriones comerciales en Madrid se distribuye de la siguiente manera: los anfitriones que tienen entre dos y cinco anuncios, representan al $21,08 \%$ de los anfitriones y controlan el 32,39\% de los anuncios; aquellos que tienen entre seis y 20 anuncios, representan al $2,66 \%$ de los anfitriones y controlan el 14,19\% de los anuncios, mientras los anfitriones con más de 20 ofertas en la plataforma solo representan el $0,47 \%$ de los anfitriones pero controlan el 
$8,41 \%$ de los anuncios (ver tabla 1). De hecho, 34 anfitriones tienen un total de 1.027 anuncios en la plataforma. Por el contrario, los anfitriones del modelo p2p (esporádicos y permanentes), si bien representan a un 75,79\% de los anfitriones, tan solo controlan el $45,01 \%$ de los anuncios. Por lo tanto, se puede afirmar que los anfitriones profesionales e inversores, que realizan la actividad de manera comercial y que no cumplen los principios de la economía colaborativa, aunque solo representan a una minoría de los anfitriones de Airbnb en Madrid, controlan la mayoría de los anuncios de hospedaje de la plataforma.

Tabla 1: Distribución de los anuncios en Airbnb según la cantidad de anuncios que tiene cada anfitrión.

\begin{tabular}{|c|c|c|c|c|c|}
\hline & Un anuncio & $\begin{array}{c}\text { Entre 2 y 5 } \\
\text { anuncios }\end{array}$ & $\begin{array}{c}\text { Entre 6 y } \\
20 \text { anuncios }\end{array}$ & $\begin{array}{c}\text { Más de 20 } \\
\text { anuncios }\end{array}$ & $\begin{array}{c}\text { Total anun- } \\
\text { cios }\end{array}$ \\
\hline Anfitriones & 5.493 & 1.528 & 193 & 34 & 7.248 \\
\hline$(\%)$ & $75,79 \%$ & $21,08 \%$ & $2,66 \%$ & $0,47 \%$ & $100 \%$ \\
\hline Anuncios & 5.493 & 3.953 & 1.732 & 1.027 & 12.205 \\
\hline$(\%)$ & $45,01 \%$ & $32,39 \%$ & $14,19 \%$ & $8,41 \%$ & $100 \%$ \\
\hline
\end{tabular}

Fuente: elaboración propia con datos de Inside Airbnb (de abril de 2017).

La presencia de anfitriones comerciales con múltiples anuncios en Airbnb es uno de los principales focos de conflicto en torno a la actividad de la plataforma, lo que ha sido señalado en múltiples investigaciones (Gil, 2018; Wegmann \& Jiao, 2017; Red2Red Consultores, 2017; CBRE Hotels' Americas Research, 2017; Merante y Horn, 2016; Lee, 2016; Marqusee, 2015). En dichas investigaciones se diferencia entre anfitriones con un único anuncio (modelo p2p) y anfitriones con múltiples anuncios (modelo comercial). Pero dicho análisis es insuficiente para conocer en profundidad la realidad y efectos de Airbnb en una ciudad. Esto se debe a que se dan casos de anfitriones con un único anuncio, que alquilan los 365 días del año, y que por lo tanto responden al modelo comercial y no cumplen los principios de la economía colaborativa.

Para determinar si un anfitrión con un único anuncio en Airbnb responde al modelo p2p o al modelo comercial, se debe incluir en el análisis la variable "disponibilidad". Es decir, cuántos días al año está disponible el hospedaje en la plataforma. Si una vivienda se alquila los 365 días al año en Airbnb, dicha vivienda no puede funcionar como la primera residencia del anfitrión, ni por lo tanto cumplir el principio de "bien temporalmente ocioso" de las economías colaborativas. La cantidad de días que una vivienda cumple la función de bien ocioso depende del uso que haga cada persona de su vivienda. Algunas administraciones públicas, con fines regulatorios, han optado por establecer un máximo de días al año en que se puede realizar esta actividad sin que sea considerada como una actividad comercial sino como una actividad propia del campo de las economías colaborativas. Aunque varía de una ciudad a otra, los periodos máxi- 
mos permitidos para dicha actividad suelen variar entre 60 y 120 días, siendo la cifra de 90 días una de las más frecuentes (Gil, 2018). De hecho, la regulación del propio Ayuntamiento de Madrid $^{2}$ distingue entre el modelo p2p y el modelo comercial, y establece el límite de 90 días al año para que la actividad sea considerada como de "economía colaborativa" (y no requiera de las licencias ni cumplimiento de la normativa urbanística que requiere la actividad de los anfitriones profesionales e inversores). Por lo tanto, para los siguientes análisis, se utilizará la disponibilidad de "90 días" como la variable que permita diferenciar entre la actividad del modelo p2p y la actividad comercial.

De esta manera, se observa que en Madrid tan solo el 11,26\% de los anuncios en Airbnb corresponden a formas de hospedaje entre particulares. Por el contrario, el $88,74 \%$ de los anuncios en la ciudad lo controlan anfitriones profesionales e inversores. Desde esta perspectiva, se puede afirmar que la actividad de Airbnb en Madrid no se articula sobre formas de hospedaje del campo de las economías colaborativas, sino sobre actores especializados que controlan el sector (inmobiliarias, empresas especializadas y grandes y medianos propietarios).

Tabla 2: Distribución de los anuncios en Airbnb según el tipo de anfitrión.

\begin{tabular}{|c|c|c|c|c|c|c|}
\hline & \multirow{2}{*}{$\begin{array}{l}\text { Anfitriones } \\
\text { particulares } \\
\text { (economía } \\
\text { colaborati- } \\
\text { va) }\end{array}$} & \multicolumn{4}{|c|}{ Anfitriones profesionales } & \multirow{2}{*}{$\begin{array}{c}\text { Total } \\
\text { anuncios }\end{array}$} \\
\hline & & $\begin{array}{c}\text { Un anun- } \\
\text { cio }\end{array}$ & $\begin{array}{l}\text { Entre } 2 \text { y } \\
5 \text { anun- } \\
\text { cios }\end{array}$ & $\begin{array}{l}\text { Entre } 6 \text { y } \\
20 \text { anun- } \\
\text { cios }\end{array}$ & $\begin{array}{l}\text { Más de } 20 \\
\text { anuncios }\end{array}$ & \\
\hline Anuncios & 1.374 & 4.119 & 3.953 & 1.711 & 1.048 & 12.205 \\
\hline$\%$ & $11,26 \%$ & $33,75 \%$ & $32,38 \%$ & $14,02 \%$ & $8,59 \%$ & $100 \%$ \\
\hline
\end{tabular}

Fuente: elaboración propia con datos de Inside Airbnb (de abril de 2017).

Para profundizar en el fenómeno de Airbnb en Madrid y sus efectos, también se debe analizar el volumen total de la actividad que se articula sobre la plataforma. Para ello se analizará el volumen total de pernoctaciones que se pueden contratar en Airbnb. Es decir, el volumen de noches de hospedaje que se ofertan en Airbnb a lo largo del año. En Madrid se ofertan al año más de dos millones y medio de noches de hospedaje en Airbnb. De estas, tan solo un 4.3\% responden al modelo de hospedaje entre particulares, frente al $95.7 \%$ que responden al modelo de VUT comerciales (ver tabla 3). Por lo tanto, desde la perspectiva del volumen que genera la actividad de hospedaje en Airbnb, el porcentaje de la actividad que responde a formas de economía colaborativa es

${ }^{2}$ https://diario.madrid.es/blog/notas-de-prensa/aprobado-el-plan-especial-para-la-regulacionde-los-alojamientos-turisticos/

EMPIRIA. Revista de Metodología de Ciencias Sociales. N. ${ }^{\circ} 41$ septiembre-diciembre, 2018, pp. 15-32. ISSN: 1139-5737, DOI/empiria.41.2018.22602 
minoritario en la ciudad de Madrid.

Tabla 3: Distribución de las noches de hospedaje ofertadas en Airbnb según el tipo de anfitrión.

\begin{tabular}{|c|c|c|c|c|c|c|}
\hline & $\begin{array}{c}\text { Anfitriones } \\
\text { particulares } \\
\text { (economía } \\
\text { colaborati- } \\
\text { va) }\end{array}$ & $\begin{array}{c}\text { Un anun- } \\
\text { cio }\end{array}$ & $\begin{array}{c}\text { Entre 2 y } \\
5 \text { anun- } \\
\text { cios }\end{array}$ & $\begin{array}{c}\text { Entre 6 y } \\
20 \text { anun- } \\
\text { cios }\end{array}$ & $\begin{array}{c}\text { Más de } \\
20 \text { anun- } \\
\text { cios }\end{array}$ & $\begin{array}{c}\text { Total } \\
\text { anuncios }\end{array}$ \\
\hline $\begin{array}{c}\text { Noches de } \\
\text { hospedaje }\end{array}$ & 117.905 & 1.105 .207 & 872.529 & 404.233 & 245.573 & 2.745 .447 \\
\hline$(\%)$ & $4,29 \%$ & $40,26 \%$ & $31,78 \%$ & $14,72 \%$ & $8,94 \%$ & $100 \%$ \\
\hline
\end{tabular}

Fuente: elaboración propia con datos de Inside Airbnb (de abril de 2017).

En resumen y como se ha visto en los análisis previos, la mayoría de anfitriones de Airbnb en Madrid responden a personas que tan solo tienen un anuncio en la plataforma. Aun así, los que tienen múltiples anuncios controlan la mayoría del mercado. Al diferenciar entre anfitriones particulares que hospedan bajo formas de economía colaborativa y anfitriones comerciales, se observa que la diferencia es aún mayor, y que los particulares tan solo representan un pequeño segmento del mercado. Además, al analizar el volumen total de la actividad en la plataforma (las pernoctaciones), se observa que el porcentaje del mercado que responde a formas de economía colaborativa representa menos del 5\% del mercado. En general, se puede afirmar que la actividad de Airbnb en Madrid se articula sobre actores comerciales y especializados que controlan la mayoría del mercado, que no responden a formas de economía colaborativa sino a formas de economía tradicional basadas en la obtención de rentas inmobiliarias.

Desde la perspectiva de la turistificación y los efectos de Airbnb sobre el mercado de vivienda, los resultados obtenidos son alarmantes. Si la actividad de Airbnb en Madrid se articula sobre un modelo comercial, es muy probable que a través de Airbnb en Madrid se esté produciendo un efecto de sustitución de vivienda residencial por vivienda para turistas. De esta manera, nos tenemos que preguntar ¿cuántas viviendas se han extraído del mercado del alquiler de larga duración y se han convertido en viviendas para turistas en Airbnb?

Para realizar dicha estimación, se van a seleccionar todos los anuncios de Airbnb que se alquilan como "alojamiento entero"3 durante más de 90 días al año. Es decir, toda la oferta de alquiler de pisos enteros de anfitriones profesionales e inversores. Como se observa en el gráfico 1, en Madrid potencialmente se habrían extraído del mercado de alquiler 6.034 viviendas. Estas viviendas re-

${ }^{3}$ En Airbnb existen tres tipos de alojamientos: alojamiento entero, habitación privada y habitación compartida. 
presentan un 76,04\% del total de los alojamientos enteros que se ofertan en Airbnb, ya que solo 1.901 de los pisos ofertados -un 23,96\%- se estarían alquilando bajo los principios de la economía colaborativa. Es decir, un 76,04\% de los alojamientos enteros que se alquilan en Airbnb se podrían estar alquilando como vivienda residencial. Si en Madrid se aprobase la legislación de pisos turísticos de la ciudad de San Francisco ${ }^{4}$, el alquiler de estas viviendas en Airbnb quedaría prohibido, y la gran mayoría volverían al mercado del alquiler residencial de larga duración (Lee, 2016; Wegmann y Jiao, 2017). Además, esta sustitución de vivienda residencial por vivienda para turistas tiene un efecto mayor en el Distrito Centro de la ciudad, ya que un 50.84\% de las ofertas de Airbnb en Madrid se encuentran en dicho distrito. Como se observa en el gráfico, solo en Distrito Centro se habrían transformado 3.758 viviendas residenciales en alojamientos para turistas, y tan solo 1.043 de los alojamientos enteros que se alquilan en Airbnb en el Distrito Centro estarían cumpliendo los principios de las economías colaborativas.

\section{Gráfico 1: Sustitución de vivienda residencial por pisos para turistas en Airbnb.}

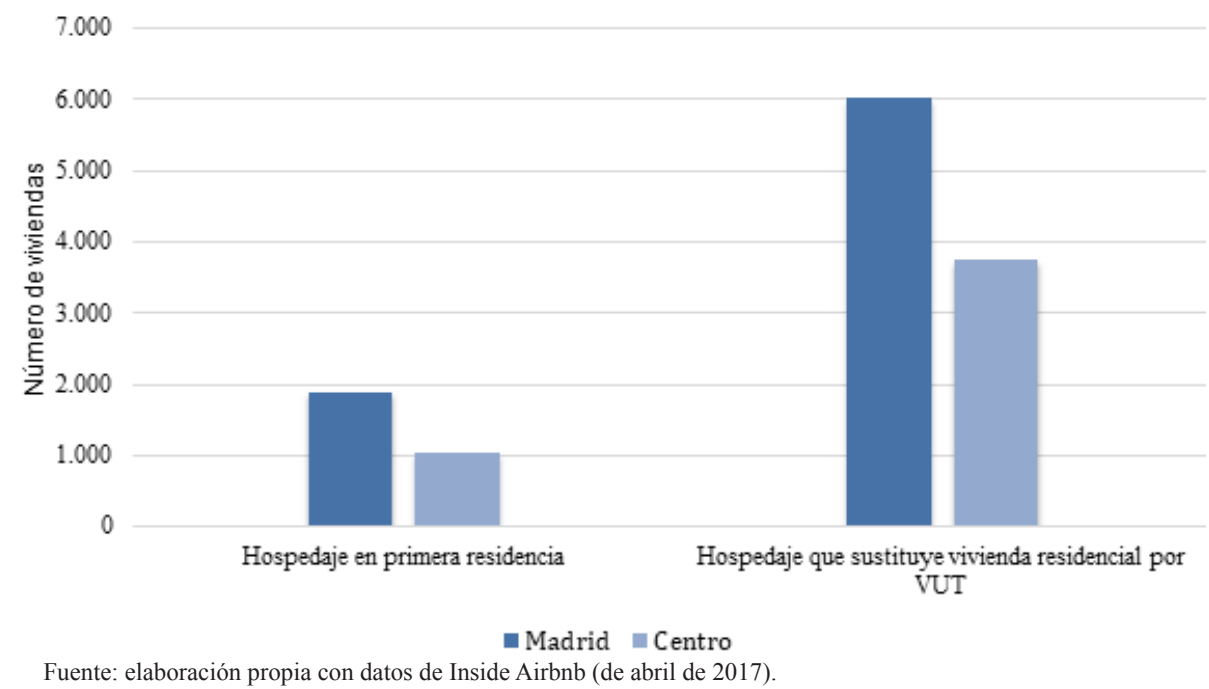

Este efecto de sustitución de vivienda residencial por vivienda para turistas, en el distrito de Madrid más afectado por la turistificación, amplía de forma exponencial los efectos de la turistificación, ya que tiene un fuerte impacto sobre los precios de los alquileres, los desplazamientos de la población local, la convivencia en el barrio y la transformación del comercio local.

${ }^{4}$ La regulación de San Francisco está siendo de las más eficaces del mundo para impedir que a través de plataformas como Airbnb se sustituya vivienda residencial por viviendas para turistas (Gil, 2018). 
En definitiva, a día de hoy, en la mayoría de ciudades globales no se puede hablar de turistificación sin hablar de crisis de vivienda -y viceversa-. Son dos procesos que van de la mano, algo que también se refleja en la propia composición de los grupos y colectivos que organizan formas de resistencia contra estas formas de transformación neoliberal de la ciudad, como veremos a continuación.

\section{RESISTENCIAS CONTRA LA CIUDAD TURÍSTICA: EL CASO DE MADRID}

Como hemos presentado en los apartados anteriores, los principales conflictos generados por la turistificación de áreas del centro de ciudades europeas permiten superar la barrera de una perspectiva acrítica de la economía turística o de sus efectos positivos, sumergiéndonos en el debate histórico del derecho a la ciudad. Nos referimos al derecho de acceso, uso y disfrute de la ciudad o, en otras palabras, al derecho de participar en la producción de lo urbano. Vistos como sujetos políticos y sociales, los movimientos sociales urbanos tienen la capacidad de sacar a la luz las consecuencias negativas de las políticas urbanísticas neoliberales proponiendo alternativas, llevando a cabo acciones o visibilizando las desigualdades e injusticias que tienen lugar en la ciudad (Chatterton and Pickerill, 2010). Por esta razón, las luchas vecinales son un intento de contrarrestar los efectos del neoliberalismo así como un inmejorable punto de partida para generar nuevas condiciones sociales en los barrios mediante la extensión a múltiples niveles de la solidaridad y el apoyo mutuo. Este activismo urbano no sólo muestra las incoherencias y contradicciones del capitalismo (Holloway, 2010; Harvey, 2014), sino que también posibilita la creación de redes de ciudadanía alternativas que se enfrentan a la actual hegemonía neoliberal.

En el caso de la ciudad de Madrid, todo el área central de la ciudad sufre actualmente una nueva burbuja especulativa, originada por un turismo que ha sido visto como motor económico "durante la crisis". Este escenario ha provocado en los dos últimos años un aumento de la protesta organizada. Así, de las distintas luchas organizadas que se están dando en la ciudad, nos queremos detener en el colectivo llamado "Lavapiés, ¿dónde vas?" $(\mathrm{LdV})^{5}$ y en la alianza de colectivos sociales que han presentado una campaña a favor de una moratoria turística en la ciudad de Madrid.

En primer lugar, nos referiremos a la campaña iniciada por el colectivo $\mathrm{LdV}$, puesto que fue el primero en dotarse de herramientas y repertorios de acción frente a la turistificación en Madrid. En un primer momento, desde este colectivo vecinal, se llevó a cabo un amplio diagnóstico participativo de su propio barrio cuando ya se habían sufrido más de 15 años de políticas gentrificadoras (Sequera, 2013), incluyendo una crisis económica. Esta manera de trabajar colectivamente implicó no sólo una sistematización de informes y estudios sobre el barrio, sino también la creación de una fuerte red en el barrio capaz de seguir

\footnotetext{
${ }^{5}$ https://lavapiesdondevas.wordpress.com/
} 
avanzando en la lucha. De esta forma, "hacer comunidad" (desde asociaciones de padres y madres, sociedad civil o activistas anticapitalistas) fue un primer paso para trazar un mapa del entramado neoliberal, señalando los espacios más conflictivos o los espacios de oportunidad que aún existen en el barrio.

La segunda fase, y a diferencia de otras acciones más disruptivas desplegadas en algunos barrios de Barcelona (Sequera y Nofre, 2018), este grupo activista ha buscado caricaturizar los efectos más negativos del turismo en la zona. Para ello, llevaron a cabo cuatro acciones: 1) "Lavapiés se subasta", un ejercicio irónico en que se subastaban a los vecinos susceptibles de ser expulsados: "subastamos niñas y niños, vecinas y vecinos de todas las edades, autóctonos y migrantes, feministas, comerciantes, tenderos y tenderas, activistas, cañas a un euro, pisos compartidos, carritos de la compra... Trae todo lo que ya no tiene lugar en este nuevo barrio turístico y elitizado y véndelo al mejor postor"; 2) "El destierro del vecino", celebrado en la Fiesta de Carnaval y que trataba de hacer sátira mediante el entierro de un vecino que "se va al otro barrio"; 3) "Una manifestación por los derechos del turista", donde los vecinos disfrazados de turistas y paseando maletas demandaban que el barrio entero se adaptase a sus necesidades como visitantes; y 4) se trazó un mapa de los pisos de Airbnb (titularidades de los mismos, inmobiliarias que los gestionan, vecinos amenazados por acoso de la inmobiliarias, etc.).

La tercera etapa, a mediados de 2017, conocida por el colectivo como "políticas de contención" consistieron avanzar desde el diagnóstico compartido de la primera fase a una batería de propuestas tangibles. Conscientes de la dificultad de revertir las políticas gentrificadoras que han dejado una fuerte impronta en Lavapiés durante casi 20 años (Sequera, 2013), los vecinos decidieron afrontar estos problemas desde una perspectiva poliédrica: cuando hablan de contención, se refieren ante todo a paralizar esas políticas o, al menos, ralentizar su velocidad y virulencia. Se refieren a la capacidad de frenar todas las transformaciones potenciales que podrían ocurrir o con las que conviven en el presente y que aún no están consolidadas. Con el fin de pensar y trabajar colectivamente contra tales políticas, prepararon dos líneas de acción: (i) idear medidas públicas que fueran capaces de paralizar algunos de estos procesos, para luego forzar al Ayuntamiento a implementar tales políticas; (ii) a través de la auto-organización, tener capacidad autónoma para la toma de decisiones en el barrio, con la puesta en marcha de un "Plan Sostenible de Lavapiés" que, entre otras cosas, paralizaría la construcción de nuevos hoteles, la turistificación y los desahucios.

Una de las acciones más importantes realizadas por el colectivo en dicho contexto, fue la denuncia pública de pisos turísticos controlados por grandes tenedores de viviendas de uso turístico (VUT). Lavapiés, ¿dónde vas?, junto a otros colectivos de vivienda de la ciudad como PAH Centro, el Sindicato de Inquilinas e Inquilinos de Madrid y la ILP de Vivienda de Madrid, denunciaron el caso de cuatro empresas profesionales de alquiler de VUT. En concreto denunciaron "18 edificios residenciales, de los cuales 10 están dedicados íntegramente a viviendas o apartamentos turísticos, teniendo el resto más del 50\% de sus vi- 
viendas destinadas a esa actividad" y que en total suman 203 viviendas de uso turístico en el barrio (todas ellas pertenecientes a estas cuatro empresas). Entre estas destaca la empresa Friendly Rentals Madrid ${ }^{7}$, que según denunciaron tiene más de 177 pisos turísticos en la ciudad de Madrid. Como señala el colectivo, detrás de la empresa se encuentra Wyndham Worldwide ${ }^{8}$, el primer grupo hotelero del mundo, que cuenta con cerca de 8.000 hoteles bajo 15 marcas en 153 países y 6 continentes, que totalizan más de medio millón de habitaciones. Sin lugar a duda, este tipo de hospedaje no responde a los principios de la economía colaborativa y su modelo de negocio se basa en convertir vivienda residencial en VUT.

El acto de denuncia tuvo una gran repercusión mediática9 ${ }^{9}$, e instaba a los poderes públicos a sancionar a dichas empresas por el incumplimiento de la normativa. Además, hacían publica una denuncia-tipo con la que cualquier vecino de Madrid podría denunciar ante el Ayuntamiento las VUT ilegales. Un mes más tarde, ante la inactividad del Ayuntamiento, el colectivo volvía a lanzar en redes la campaña \#Manueladinosalgo ${ }^{10}$ y \#1mesmas100viviendasmenos. Bajo el lema "18 denuncias, cero respuestas" y "1 mes más, 100 viviendas menos", el colectivo instaba al Ayuntamiento a no quedarse de manos cruzadas y a sancionar a las empresas a las que habían denunciado un mes antes. Como se mostraba en algunas publicaciones del colectivo, la tensión con el "ayuntamiento del cambio" se hacía cada vez más evidente: "Esconderse no es una opción maj@s, las denuncias hay que tramitarlas y darles respuesta, porque mientras siguen desapareciendo viviendas, subiendo los precios y cerrando comercios. Todo muy poco Ahora Madrid, ¿no?"

Si bien hasta el momento las principales acciones habían sido de diagnóstico y de presión a la institución para que sancione los abusos e ilegalidades, el objetivo de estas acciones no se limitaba a una actuación administrativa más restrictiva o garantista. El objetivo era cuestionar el modelo de ciudad que está generando la creciente turistificación, y la articulación de los procesos de acumulación de capital y sus nuevas burbujas inmobiliarias, que pliegan al conjunto de las ciudades a intereses cortoplacistas y especulativos, en detrimento de las personas y la calidad de vida ${ }^{11}$.

Volviendo a la fase de "políticas de contención" que hemos subrayado anteriormente, ésta ha cobrado mayor relevancia a principios de 2018. El 16 de enero de 2018, el colectivo Lavapiés ¿dónde vas? junto a la Federación Regional de Asociaciones de Vecinos de Madrid (FRAVM), la Coordinadora de Asociaciones de Vecinos de Centro, Ecologistas en Acción, la Plataforma de Afectados por la Hipoteca (PAH) y el Sindicato de Inquilinas e Inquilinos de Madrid, realizaban

${ }^{6}$ https://lavapiesdondevas.files.wordpress.com/2017/09/edificios-viviendas-turisticas.pdf

7 https://www.friendlyrentals.com

8 www.wyndhamworldwide.com/

9 En la entrada en Facebook que publicaron el 2 de octubre del 2017 recopilan los enlaces a más de una docena de medios que recogieron la denuncia en sus noticias

${ }^{10}$ Que fue tendencia en Twitter el 30 de octubre en Madrid.

${ }^{11}$ https://www.facebook.com/lavapiesdonde/posts/1494484227335150 
una rueda de prensa conjunta ante la sede del Gobierno de la Comunidad de Madrid en la Puerta del Sol. En dicha rueda de prensa los colectivos sociales y vecinales demandaban a las autoridades la elaboración de un Plan Especial de Ordenación Turística para la ciudad de Madrid. Además, demandan que este plan debería realizarse mediante la apertura de "un proceso participativo abierto a todos los agentes interesados en el diseño de un plan de desarrollo turístico sostenible, esto es, compatible con el derecho a la ciudad de los y las habitantes de Madrid", como señalaron en la nota de prensa. Asimismo, también exigían una moratoria a la concesión de licencias para todo tipo de plazas turísticas en toda la ciudad hasta la aprobación de dicho plan.

A partir de este momento se observa un cambio en la lucha contra la turistificación en la ciudad de Madrid. Por un lado, se pasa de acciones destinadas a visibilizar el problema - concienciar a los ciudadanos, introducir el conflicto de la turistificación en la agenda mediática y política, y de presión a las instituciones para que intervengan a través de sanciones- a una fase propositiva. Son los colectivos sociales y vecinales los que se adelantan a la administración y proponen medidas concretas ante el problema de la turistificación: un Plan Especial de Ordenación Turística, acompañado de una moratoria de licencias mientras se elabora el Plan. Por otro lado, también se observa un cambio en la composición de los colectivos movilizados en torno a la turistificación. Si Lavapiés ¿dónde vas? había sido el principal grupo movilizado en torno a esta cuestión, posteriormente se sumarían colectivos de vivienda como la $P A H$ o el Sindicato de Inquilinas $e$ Inquilinos, la FRAVM y la Coordinadora de Asociaciones de Vecinos de Centro o incluso grupos como Ecologistas en Acción.

De forma paralela a esta paulatina incorporación de otros colectivos a la lucha contra la turistificación, el colectivo Lavapiés ¿dónde vas? lanzaba la propuesta "Moratoria turística en el centro de Madrid" en la plataforma Decide Madrid $^{13}$, plataforma de participación ciudadana desarrollada por el Ayuntamiento de Madrid en la que se pueden realizar "Propuestas Ciudadanas" que de ser votadas por el $1 \%$ del censo, debe ser sometida a consulta ciudadana (referéndum).

12 http://moratoria.eu/

13 https://decide.madrid.es/proposals/20657-moratoria-turistica-en-el-centro-de-madrid (última visita: $26 / 7 / 2018$ ) 


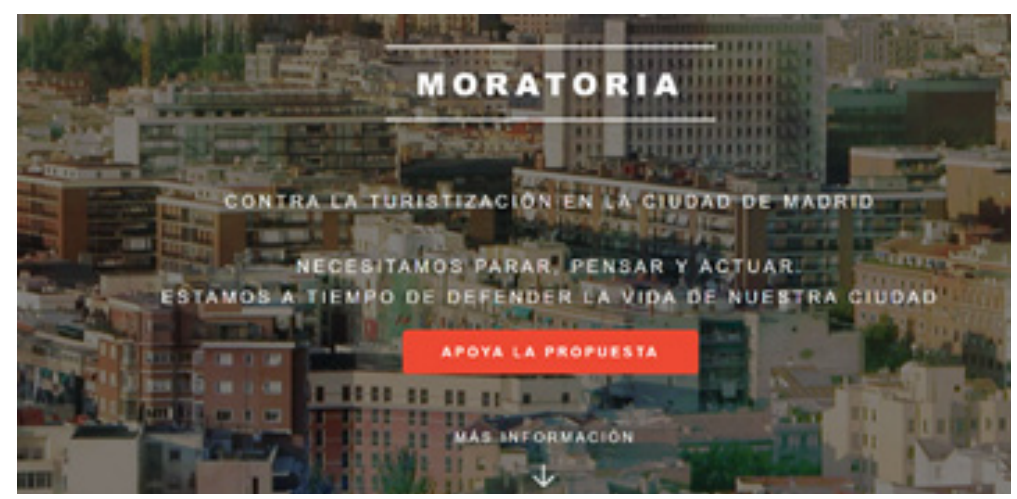

Fuente: moratoria.eu

La propuesta colectiva proponía la redacción de un Plan Especial de Ordenación Turística (PEOT) de la Ciudad y una moratoria en la concesión de licencias tanto de pisos turísticos como de hoteles durante el proceso. A diferencia de la propuesta del Ayuntamiento, esta propuesta insiste en que la elaboración del PEOT sea participativo, como se señala en la propia moratoria. Además, la propuesta de moratoria no sería en exclusiva para zonas del centro de la ciudad, sino que incluye distritos de la periferia madrileña como Puente de Vallecas, Usera, Carabanchel o Latina. El efecto de la moratoria en Decide Madrid fue inmediato: pese a las claras limitaciones de un modelo de participación on-line como DecideMadrid, la propuesta es la segunda propuesta de la plataforma con más apoyos. La buena acogida de la propuesta refleja que los problemas derivados de la turistificación cuentan con un amplio apoyo ciudadano, fruto en gran parte de las campañas de visibilizarían y difusión del problema realizada por colectivos como Lavapiés ¿dónde vas?

Esta propuesta de moratoria recoge una serie de diagnósticos colectivos sobre turistificación realizados durante el último año. Titulado "moratoria o barbarie", busca señalar algunos de los efectos que está teniendo la turistificación en la ciudad de Madrid: 10.000 vecinos menos en Centro; 73.878 plazas turísticas en Centro, donde residen 149.718 habitantes (1 plaza turística por cada 2 habitantes); más del 39,9\% y del $39.1 \%$ de subida de los precios de los alquileres en Vallecas y Centro; 4,3\% de noches de alquiler en Airbnb bajo un modelo de economía colaborativa, y un 95,7\% de alquiler en Airbnb de pisos turísticos que se alquilan de manera profesional. 

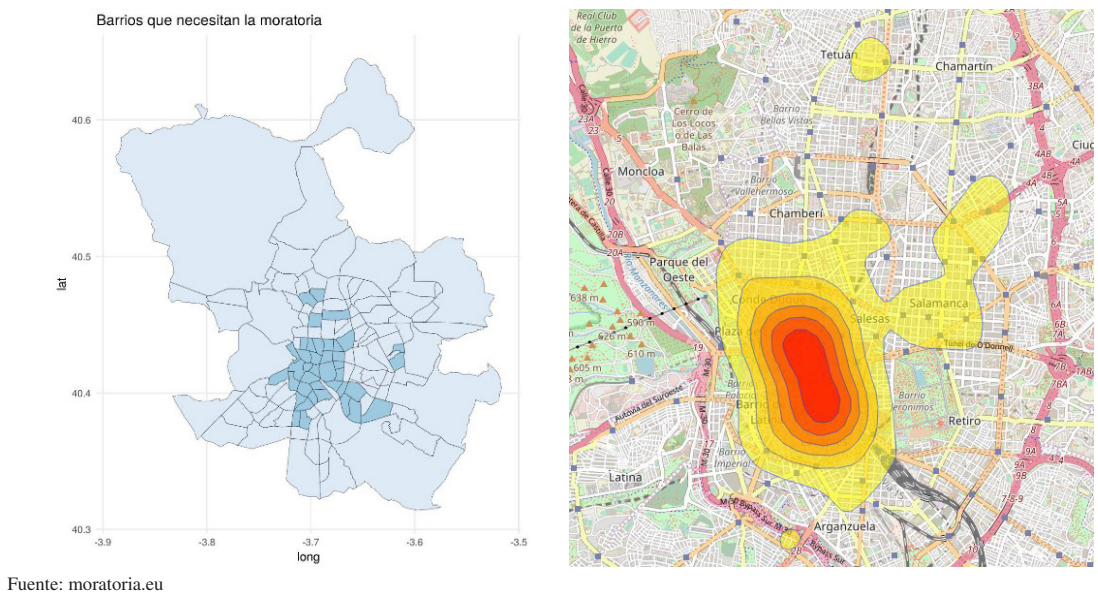

La propuesta de moratoria señala los problemas que el monocultivo turístico genera y los efectos que tiene sobre la ciudad. Ésta insiste en las desigualdades que el turismo produce en las ciudades: si bien los beneficios económicos para las empresas del sector son importantes, se trata de un sector que precariza al trabajo. Además, los defensores de esta moratoria consideran que este tipo de economía turística introduce dinámicas en la ciudad que favorecen la acumulación de capital pero reducen el bienestar y limita los usos que la ciudadanía puede hacer de la ciudad. Todo ello genera abruptos procesos de transformación de la ciudad, en un contexto en que el rápido crecimiento del turismo ha ido acompañado de una lenta actuación institucional, poniendo en cuestión la capacidad de la administración pública por si sola para hacer frente a este fenómeno.

Ante este diagnóstico, la propuesta de moratoria plantea propuestas concisas. Por un lado, pone en manos de la ciudadanía que dicha moratoria se lleve a cabo, ya que sus apoyos y votos son necesarios en el proceso. Pero además la propia moratoria incide en que el PEOT sea participado por los ciudadanos, lo que supone que la propia ciudadanía pueda decidir el modelo de ciudad que desean. También recoge una serie de medidas concretas que se deberían llevar a cabo y que la administración no ha realizado hasta el momento, como propuestas para reducir el monocultivo turístico.

\section{CONCLUSIONES}

Como hemos podido ver, el impacto del turismo es uno de los nuevos desafíos a la hora de repensar la ciudad, tanto desde el ámbito académico como desde el activismo. Contemplamos cómo, a pie de calle, la producción en la ciudad ha dado un nuevo giro, tomando al turista como principal consumidor. 
Este contexto está atravesado por el surgimiento y rápido desarrollo durante los últimos años de la plataforma digital Airbnb, que se ha convertido en un símbolo de las nuevas formas de turistificación de la ciudad. Bajo la promesa de los impactos positivos que iban a propagar las nuevas economías colaborativas, el hospedaje a través de plataformas como Airbnb iba a generar modelos de ciudad más justos, sociales y medioambientales, que impulsaran el comercio local y formas de redistribución económica -para que los particulares pudieran obtener ingresos extra gracias al turismo en su ciudad-. Pero como hemos visto en este estudio, Airbnb en Madrid está controlado por actores económicos profesionales (inmobiliarias, empresas especializadas, grandes y pequeños propietarios), cuya actividad consiste en extraer vivienda del mercado del alquiler para introducirla en Airbnb, lo que pone en evidencia el mito de los beneficios de este tipo de economías para la ciudad.

Estos modelos de turistificación también han generado nuevos ciclos de resistencia. Como hemos mostrado anteriormente, los movimientos urbanos contra la turistificación en Madrid y la coordinación de colectivos bajo la lucha contra la turistificación se mantiene en el tiempo y se formaliza, llegando incluso a convertirse en actores políticos clave y reconocidos por la propia administración. Además, tras la llegada al poder del municipalismo en Madrid en Mayo de 2015 la línea de acción parece haberse desdoblado: la de ejercer como grupo de presión (conflictivo) frente al Ayuntamiento, buscando soluciones parciales que paralicen los efectos de la turistificación; y por otro lado, desde la autonomía, generando sus propios diagnósticos y alternativas, que promuevan políticas sostenibles e imaginativas para luchar contra la especulación.

Esas posiciones antagónicas adoptadas por los diferentes actores y grupos sociales afectados y/o involucrados en la reciente expansión del turismo urbano en el centro de muchas ciudades europeas, requieren urgentemente afrontar y abordar la interacción compleja y no lineal entre el 'derecho a la ciudad' y una convivencia inclusiva en 'barrios turistificados' en conflicto.

\section{BIBLIOGRAFIA}

Airbnb. (2018). Informe sobre la vivienda en Madrid. Airbnb.

Alex Marqusee. (2015). Airbnb and San Francisco: Descriptive Statistics and Academic Research. San Francisco Planning Department.

Ashworth, G. \& Page, S. J. (2011). Urban tourism research: Recent progress and current paradoxes. Tourism Management 32(1): 1-15. doi:10.1016/j.tourman.2010.02.002

Baptista, L. (2005). Territórios lúdicos (e o que torna lúdico um território): ensaiando um ponto de partida. Fórum Sociológico, 13/14: 47-58.

Botsman, R., y Rogers, R. (2011). What's mine is yours: how collaborative consumption is changing the way we live. London: Collins.

CBRE Hotels’ Americas Research. (2017). Hosts with Multiple Units- A Key Driver of Airbnb Growth: A Comprehensive National Review Including a Spotlight on 13 U.S. Markets. CBRE. 
Chatterton, P., \& Pickerill, J. (2010). Everyday activism and transitions towards postcapitalist worlds. Transactions of the institute of British Geographers, 35(4), 475490.

Cocola-Gant, A. (2016). Holiday Rentals: The New Gentrification Battlefront. Sociological Research Online, 21(3), 10

Colomb, C., \& Novy, J. (Eds.). (2016). Protest and resistance in the tourist city. Routledge.

Conti, G., \& Perelli, C. (2007). Governing tourism monoculture: Mediterranean mass tourism destinations and governance networks. Tourism and Politics. Global Frameworks and Local Realities, Amsterdam, Elsevier, 235-261.

Cummings, J. (2015). Confronting favela chic: The gentrification of informal settlements in Rio de Janeiro, Brazil. Global gentrifications: Uneven development and displacement, 81-99.

Gil, J. (2018). ¿Cómo regular el hospedaje entre particulares? Criterios de regulación utilizando el caso de Airbnb. En En Ana María de la Encarnación (Ed.): La regulación del alojamiento colaborativo. Viviendas de uso turístico y alquiler de corta estancia en el Derecho español. Thompson-Reuters Aranzadi.

González, S., \& Waley, P. (2013). Traditional retail markets: The new gentrification frontier? Antipode, 45(4), 965-983

Gordo, A., Rivera, J. y Cassidy, P. (2017). "La economía colaborativa y sus impactos sociales en la era del capitalismo digital", en Cotarelo, R. y Gil, J. (Coords.) (2017). Ciberpolítica: gobierno abierto, redes, deliberación, democracia. Madrid: Instituto Nacional de Administración Pública

Gotham, K. F. (2005). Tourism gentrification: The case of new Orleans' vieux carre (French Quarter). Urban Studies, 42(7), 1099-1121.

Gottlieb, C. (2013). Residential Short-Term Rentals: Should Local Governments Regulate the'Industry'?. Planning \& Environmental Law, 65(2), 4-9.

Hadfield, P. (2006). Bar Wars: Contesting the Night in Contemporary British Cities. Oxford: Oxford University Press.

Hadfield, P. (ed.). (2009) Nightlife and Crime: Social Order and Governance in International Perspective. Oxford: Oxford University Press

Harvey, D. (2014). Diecisiete contradicciones y el fin del capitalismo. Madrid: Traficantes de Sueños

Herzer, H., Di Virgilio, M. M., y Rodriguez, C. 2015, "Gentrification in Buenos Aires: global trends and local features". En Global gentrifications: Uneven development and displacement, 199-222.

Holloway, J. (2010). Crack capitalism. Pluto Press (UK).

Janoschka, M., \& Sequera, J. (2016). Gentrification in Latin America: addressing the politics and geographies of displacement. Urban Geography, 1-20.

Kaplan, T. (1982). Female consciousness and collective action: The case of Barcelona, 1910-1918., Signs, 7(3), 545-566.

Lee, D. (2016). How Airbnb short-term rentals exacerbate Los Angeles's affordable housing crisis: Analysis and policy recommendations. Harv. L. \& Pol'y Rev., 10, 229.

Meleo, Linda, Alberto Romolini, and Marco De Marco. "The sharing economy revolution and peer-to-peer online platforms. The case of Airbnb." International Conference on Exploring Services Science. Springer, Cham, 2016. 
Merante, M., \& Horn, K. M. (2016). Is Home Sharing Driving up Rents? Evidence from Airbnb in Boston.

Mermet, Anne-Cécile. "3 Airbnb and tourism gentrification.", en Tourism and Gentrification in Contemporary Metropolises: International Perspectives (2017): 52.

Nofre, J., Giordano, E., Eldridge, A., Martins, J. C., \& Sequera, J. (2018). Tourism, nightlife and planning: challenges and opportunities for community liveability in La Barceloneta. Tourism Geographies, 1-20.

Oskam, J., \& Boswijk, A. (2016). Airbnb: the future of networked hospitality businesses. Journal of Tourism Futures, 2(1), 22-42.

Pareja Eastaway, M., \& Simó Solsona, M. (2014). Dinámicas en el entorno construido: renovación, gentrificación y turismo. El caso de la Barceloneta. ACE : Architecture, City \& Environment, 9(26) : 201-222

Pixová, M., \& Sládek, J. (2016). "Touristification and awakening civil society in postsocialist Prague. Protest and resistance in the tourist city", En Contemporary geographies of leisure, Tourism and mobility. Routledge, London, 73-89

Red2Red Consultores. (2017, mayo). ANÁLISIS DEL IMPACTO DE LAS VIVIENDAS DE USO TURÍSTICO EN EL DISTRITO CENTRO. Ayuntamiento de Madrid: Junta Municipal de Distrito Centro.

Ritzer G. (2010) Enchanting a Disenchanted World. New York: SAGE

Sequera, J. \& Nofre, J. (2018). Urban activism and touristification in southern Europe. Barcelona, Madrid and Lisbon. In J. Roberts \& J. Ibrahim. (Eds). Left Wing Activism Today. London: Routledge. (in press)

Sequera, J. (2017). Ante una nueva civilidad urbana. Capitalismo cognitivo, habitus y gentrificación. Revista Internacional de Sociología, 75(1), 055.

Sequera, J. \& M. Janoschka (2015). Gentrification dispositifs in the historic centre of Madrid: a re-consideration of urban governmentality and state-led urban reconfiguration. In Lees, L., Shin, H.B. \& E. López-Morales (Eds.), Global Gentrifications: Uneven Development and Displacement. Bristol: Policy Press

Sequera, J. (2013). Las Políticas de Gentrificación en la ciudad neoliberal. Nuevas clases medias. Producción cultural y Gestión del Espacio Público. El caso de Lavapiés en el centro histórico de Madrid. Tesis Doctoral dirigida por Mario Domínguez Sánchez-Pinilla, Departamento de Sociología III, Universidad Complutense de Madrid

Smith, N. (1996). The new urban frontier: Gentrification and the revanchist city. Psychology Press.

Wachsmuth, D., \& Weisler, A. (2017). Airbnb and the Rent Gap: Gentrification Through the Sharing Economy.

Wegmann, J., \& Jiao, J. (2017). Taming Airbnb: Toward guiding principles for local regulation of urban vacation rentals based on empirical results from five US cities. Land Use Policy, 69, 494-501.

Wynne, D., \& O'Connor, J. (1998) Consumption and the postmodern city. Urban Studies, 35 (5-6), 841-64. doi: 10.1080/0042098984583 